Document downloaded from:

http://hdl.handle.net/10251/103513

This paper must be cited as:

Antonino-Daviu, J.; Lee, SB.; Strangas, E. (2017). Guest Editorial Special Section on Advanced Signal and Image Processing Techniques for Electric Machines and Drives Fault Diagnosis and Prognosis. IEEE Transactions on Industrial Informatics. 13(3):1257-1260. doi:10.1109/TII.2017.2690464

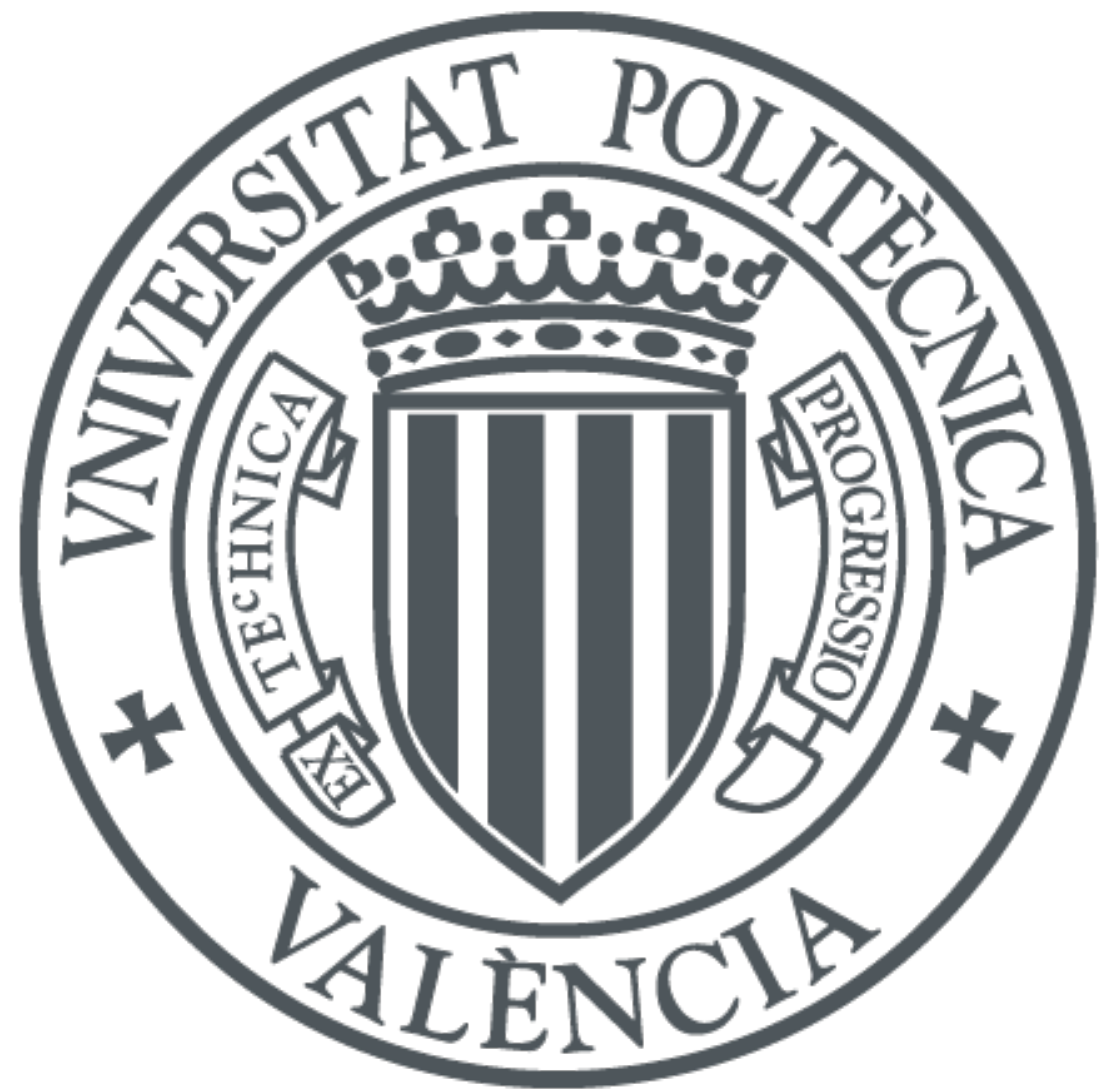

The final publication is available at

http://doi.org/10.1109/TII.2017.2690464

Copyright Institute of Electrical and Electronics Engineers

Additional Information 


\title{
Guest Editorial \\ Special Section on Advanced Signal and Image Processing Techniques for Electric Machines and Drives Fault Diagnosis and Prognosis
}

\author{
Jose A. Antonino-Daviu Senior Member IEEE, Sang Bin Lee Fellow IEEE and Elias G. Strangas Senior Member
}

IEEE

\begin{abstract}
With the expansion of the use of electrical drive systems to more critical applications, the issue of reliability and fault mitigation and condition-based maintenance have consequently taken an increasing importance: it has become a crucial one that cannot be neglected or dealt with in an ad-hoc way. As a result research activity has increased in this area, and new methods are used, some based on a continuation and improvement of previous accomplishments, while others are applying theory and techniques in related areas. This Special Section of the IEEE Transactions on Industrial Informatics attracted a number of papers dealing with Advanced Signal and Image Processing Techniques for Electric Machine and Drives Fault Diagnosis and Prognosis. This editorial aims to put these contributions in context, and highlight the new ideas and directions therein.
\end{abstract}

Index Terms-Electrical Machines, Power electronics, condition monitoring, fault detection, failure prognosis.

$\mathbf{I}^{\mathrm{N}}$

$\mathrm{N}$ recent years, the area of condition monitoring of electric machines and drives has gained a renewed dynamism. One of the reasons has been the importation of image and signal processing tools that were well-known in other scientific fields but that have had a limited application in this area. The theory and methodologies developed in the last decade proved that the use of these tools bring numerous advantages over traditional approaches. For instance, the application and optimization of advanced time-frequency transforms has enabled to greatly enhance the detection and diagnosis of many faults that occur in these machines, improving the performance of traditional industrial methods. This is critical in many industrial applications, where the reliability of the predictive maintenance techniques that are employed for the diagnosis of electric machines and drives is a crucial factor. The impact of these new technologies in this field has been so significant that they have even affected the fault prognosis area. This area has been a challenge for many motor manufacturers and end users, whose ultimate target would be to have an accurate estimation of the remaining useful life of the different machine components.

Jose Antonino-Daviu is with the Instituto Tecnologico de la Energia, Universitat Politècnica de València, 46022, Valencia, SPAIN (e-mail: joanda@die.upv.es)

Sang Bin Lee is with the Department of Electrical Engineering, Korea University, Seoul, 02841, Korea (e-mail: sangbinlee@korea.ac.kr)

Elias G. Strangas is with the Department of Electrical and Computer Engineering, Michigan State University, East Lansing, MI, 48824, USA, (email:strangas@egr.msu.edu)
This Special Section was conceived to attract recent investigations proposing innovative techniques that can enhance the fault diagnosis and prognosis in electric machines and drives. The thirteen accepted papers illustrate how the research in new methods is constantly expanding and brings new solutions for common problems that are of especial importance in diverse areas ranging from wind generation systems to electric vehicles. As an area of research, it is not yet mature, although a small but increasing number of techniques and tools is finding their way to commercial applications.

The standard, but not always used, methodology of fault diagnosis consists of a number of steps:

- Use of sensors to collect measurements related to a fault of interest,

- Extraction of features from these measurements,

- Use of these features to identify the fault and estimate the fault severity,

- Failure prognosis, i.e. estimation of the remaining useful life of the component of subsystem.

To identify the possible fault, and to estimate its severity, most often a previously developed model has to be used, based on data from a relatively large number of observations, or on an accurate physical model.

The papers in this Special Section address and offer new ideas and improvements in all these aspects. Before evaluating or assessing the contributions of each, where these works fit in this general format will be described, or how they depart from it will be described.

Firstly, features are extracted from measurements that will be subsequently used to develop a model to identify the possible faults and determine their severity. These measurements can vary between applications and the diagnosis methods used. In the papers in this Special Section, the work by Fang et al. [1] uses High Frequency (HF) resistance, and Expectation Maximization Particle Filter for the model parameter estimation, in this case for Permanent Magnet Synchronous (PMSM) machines. Dusmez et al. [2] use also a model based on measuring or estimating on-state resistance, in this case for MOSFETs. Occasionally detection is based on the forced imposition of operating conditions, so that additional features become evident; a case in point is in [3] where current harmonics are injected to vary the speed, and use the effects to determine the permanent magnet condition. A 
number of papers dealing with mechanical faults [4] [5] [6] use vibrations as the measured variables, or noise[7]. Since sensing of mechanical features is expensive and requires additional sensors, the measurement instead stator electrical quantities to determine mechanical health is used in [8] and [9], but denoising may be preferable or necessary.

To make use of the measurements and decide features, a model has to be developed and used [1], [3]. This model can be model based, statistical, [8], through approximation [2], or a linear [1] or nonlinear equation, relating it to the health state of interest. An alternative is that the model can be based on AI, mostly Neural Networks, e.g. [10]. The features used can be extracted through time domain or frequency domain analysis, and be used to determine the model parameters, either through statistical or Artificial Intelligence tools.

Additionally, these AI techniques can be used not only for determining the model, but o perform other diagnosis tasks: They can be applied directly to the raw signals [6], and with the use of multiple layers can lead directly to diagnosis. In more traditional fault diagnosis methods, Neural Networks have been used to determine coefficients, on which to base the separate characterization step. To develop these coefficients, or to identify the model structure, it is often necessary to develop and conduct tests and utilize the results to form an adequate database. This has been expensive and time consuming. In [11] where the failure identification of gearboxes is studied, Deep Neural Networks are used, based on extensive data mining, and this appears to be an important new trend.

The field of prognosis is considered central to conditionbased maintenance. Prognosis in general requires the recognition of trends in the health of a system, and a model to use. In this Special Section there are two papers that indicative of the expanding body of literature in this field. The applications discussed there are the ones that have received the greatest interest so far: power electronics, [2] and bearings, [5]. These two applications have attracted attention because in bearings faults progress relatively slowly and prognosis allows for timely maintenance, while for power electronics, an impending fault, recognized before a failure can allow reconfiguration of the control system. The work by Dusmez et al. [2] focuses on the estimation of the remaining useful lifetime (RUL) of degraded power MOSFETs, which are stressed by thermal cycling. The authors identify the relative change in on-state resistance as the fault signature. They propose a statisticsbased model to monitor the trend. They verify the accuracy of the proposed RUL estimation tool on a number of thermally aged discrete power MOSFET data. On the other hand, the paper by Singleton et al. [5] studies the relation between bearing current discharge events and bearing vibrations for bearing failure prognosis. The authors propose an extended Kalman filter and additional measurements to improve the confidence in the bearings RUL estimation. The authors make use of a novel test bed that allows the accelerated degradation of bearings due to an electrical stress placed on the bearings via shaft voltage.

Developed methods need not only be used for electrical drives. Selecting sensors and features for detection and diagnosis takes new directions in the case of buildings [12]. In this work by Li et al., the authors address the problem of optimally configuring sensors and selecting features for building FDD. On the other hand, transformer faults can be dealt with the same methods as electrical drives, both data- and model based, as is the case of the work by Ashrafian et al. [13]. This work proposes an adaptive differential protection method for power transformers. The authors make use of real-time recursive Stransform-based phasor estimation. The method includes the development of a new discrimination index to recognize inrush currents. The algorithm accuracy is validated with real data obtained in differential relays located in Western Australia.

Although the thirteen papers in this section conform for the most part to the basic structure discussed earlier, new ideas, techniques and methods have been proposed. It can be noticed that not all the recent advances in the electric motors condition monitoring area have come through the incorporation of new signal or image processing, but also optimization and enhancement of well-known methods that have been extensively used. A good example is the work by Troncoso [4]]; the author proposes an innovative method that overcomes a common deficiency of the fast Fourier Transform (FFT), the spectral leakage. This issue causes many problems when diagnosing faults in electric machines, since it leads to the appearance of spurious components due to the sampling process or to the transform itself. This complicates the discrimination between close components and may lead to severe repercussions and erroneous diagnostic conclusions. The author overcomes this problem by proposing a new approach relying on the fractional resampling. It basically consists on resampling the original sequence with an adequate combination of interpolation and decimation rates. In that way, it is possible to change the original frequency resolution to comply with the condition of being an exact multiple integer of the interest frequency, and, consequently, reduce the undesired effects of the spectral leakage. The author validates the method in a wide range of testing conditions and the results are of great potential for FFT users, since they can bring them the guidelines to avoid eventual false indications provoked by the commented issue.

This enhancement of conventional tools is combined in some works with the development and optimization of new tools in the condition monitoring area. This is the case of the work by Singh et al. [9] that proposes the application of a time-frequency tool, the Continuous Wavelet Transform (CWT), to the steady-state stator current in order to detect outer race bearing faults. The authors prove the advantages of this new approach in comparison with classical spectralbased methods. To effectively extract the fault features from the stator current signals for the faulty bearing, the authors perform a comparison between the analysis results of 2-D and 3-D wavelet scalograms for the vibration and stator current signals using the proposed methodology. On the other hand, sometimes, classical approaches are applied in novel contexts where they can provide new benefits. This is the case of the work by Picot el al. [8] that combines the Fourier series analysis and statistic tools to monitor belt looseness through the analysis of phase currents. The authors analyse relevant signatures both under steady and transient states for different speed and load conditions in order to define relevant spectral 
signatures for the monitoring. They conclude that the transient state is more appropriate for belt looseness detection because a sudden acceleration amplifies the relative belt slip.

A research line that has lived a significant advance in this area is focused on the development of new classification algorithms based on robust feature detection methods whose ultimate target is to make the diagnostic of the different faults automatic, avoiding the necessity of user intervention. In this context, some works developed methods that enhance the performance of modern feature extraction methodologies. This is the case of the work by Yang et al. [7] that proposes an approach to enhance the sparse representation method, a relatively recent method in the condition monitoring area. Although it has shown good results, this method has also some drawbacks as the necessity of a dictionary of atoms, which should be similar to the inner structure of the analyzed signal, to perform a good time-frequency analysis and feature extraction. This problem can be critical in machines under variable operating conditions, where the characteristics of the analyzed signals are changing over time, such as wind power generators. To solve the problem, a novel data-driven fault diagnosis method based on sparse representation and shiftinvariant dictionary learning is proposed. This approach is applied to the diagnosis of bearing faults in wind turbine generators. The methodology is validated both with laboratory and field data. The results prove its advantages, compared with traditional sparse representation methods, such as avoidance of the dependence of prior knowledge and the selection of proper dictionary and achievement of more effectiveness and robustness under nonstationary and strong noise conditions.

In the same line, the work by Sun et al. [[10] proposes a feature learning scheme, named convolutional discriminative feature learning, for the diagnosis of different induction motor failures. One of the main novelties of the method is that it can learn features directly from raw vibration data, avoiding the intermediate steps. It is based on a smart architecture relying on back propagation-based Neural Networks for filter weights learning and on a convolutional pooling structure to derive the invariant features. The system makes ulterior use of a Support Vector Machine classifier to identify the faulty condition. The proposed method proves to be robust and effective for the detection of diverse failures including broken rotor bars, rotor bending, bearing failure, stator windings fault and unbalanced rotor.

The search for new variants of artificial intelligence-based techniques for enhancing the feature detection and fault classification processes is also a common denominator in many works developed in the area. Two papers included in this Special Section are illustrative examples, such as the works by Wang et al. [11]] and by Liu et al. [6]. In the first one, the authors propose the use of a Deep Neural Networks (DNN) framework for monitoring the gearbox condition in wind turbines. The presented approach relies on SCADA data of the gearbox lubricant pressure and it is based in two stages: first, development of an accurate lubricant pressure prediction model (modelling) and, second, implementation of an exponentially weighted moving average (EWMA) control chart to derive criteria for monitoring the lubricant pressure (monitoring). In the first stage, the developed algorithm is compared with other alternatives, offering more accurate prediction results. Moreover, a benchmarking between the proposed monitoring quantity (lubricant pressure) and monitoring the gearbox oil temperature was carried out. The effectiveness of the proposed method is demonstrated by examining real cases from wind farms in China and benchmarked against the gearbox monitoring based on the oil temperature data. On the other hand, the work by Liu et al. [6] makes use of convolutional neural networks (CNN) to build a new diagnosis framework based on the characteristics of industrial vibration signals. This approach is named Dislocated Time Series CNN (DTS-CNN). The main advantage of DTS-CNN is that it is suited to act on raw data, reducing the demand of prior knowledge while enabling the automatic learning of robust features. It is especially interesting the fact that the authors rove the validity of their approach for machines operating under nonstationary conditions, a fact that confers great industrial attractive to their scheme.

The Guest Editors would like to thank all the authors who contributed to this Special Section, since their valuable works are an evidence of the dynamism and excellence that is living this research area. The Guest Editors would also like to express their gratitude to the reviewers that spend their expertise, time and effort for developing their throughout reviews of the works, providing expert advice that have served to enhance the quality of the contributions. Last but not least we would like to thank the readers of this Special Section for their interest in this research area and we sincerely hope that they will appreciate the contributions here included.

We would like to close recognizing the help, support and guidance provided both by the former and current Editor-inChiefs of the IEEE TRANSACTIONS ON INDUSTRIAL INFORMATICS, Prof. K. F. Man and Prof. Ren Luo. Thanks also go to S. McLain and L. Pattillo, TII staff, for their professional support and assistance during the whole preparation of this Special Section.

\section{REFERENCES}

[1] G. Feng, C. Lai, and N. C. Kar, "Expectation-maximization particlefilter- and Kalman-filter-based permanent magnet temperature estimation for PMSM condition monitoring using high-frequency signal injection," IEEE Transactions on Industrial Informatics, vol. 12, June 2017.

[2] S. Dusmez, M. Heydarzadeh, M. Nourani, and B. Akin, "Remaining useful lifetime estimation for power MOSFETs under thermal stress with RANSAC outlier removal," IEEE Transactions on Industrial Informatics, vol. 12, no. 3, June 2017.

[3] G. Feng, C. Lai, and N. C. Kar, "Particle-filter-based magnet flux linkage estimation for PMSM magnet condition monitoring using harmonics in machine speed," IEEE Transactions on Industrial Informatics, vol. 12, June 2017.

[4] R. de Jesus Romero-Troncoso, "Multirate signal processing to improve FFT-based analysis for detecting faults in induction motors," IEEE Transactions on Industrial Informatics, vol. 12, June 2017.

[5] R. K. Singleton II, E. G. Strangas, and S. Aviyente, "The use of bearing currents and vibrations in lifetime estimation of bearings," IEEE Transactions on Industrial Informatics, vol. 12, June 2017.

[6] R. Liu, G. Meng, B. Yang, C. Sun, and X. Chen, "Dislocated time series convolutional neural architecture: An intelligent fault diagnosis approach for electric machine," IEEE Transactions on Industrial Informatics, vol. 12, June 2017

[7] B. Yang, R. Liu, and X. Chen, "Fault diagnosis for a wind turbine generator bearing via sparse representation and shift-invariant K-SVD,' IEEE Transactions on Industrial Informatics, vol. 12, June 2017. 
[8] A. Picot, E. Fournier, J. Régnier, M. TientcheuYamdeu, J.-M. Andréjak, and P. Maussion, "Statistic-based method to monitor belt transmission looseness through motor phase currents," IEEE Transactions on Industrial Informatics, vol. 12, June 2017.

[9] S. Singh and N. Kumar, "Detection of bearing faults in mechanical systems using stator current monitoring," IEEE Transactions on Industrial Informatics, vol. 12, June 2017.

[10] W. Sun, R. Y. Rui Zhao, S. Shao, and X. Chen, "Convolutional discriminative feature learning for induction motor fault diagnosis," IEEE Transactions on Industrial Informatics, vol. 12, June 2017.

[11] L. Wang, Z. Zhang, H. Long, J. Xu, and R. Liu, "Wind turbine gearbox failure identification with deep neural networks," IEEE Transactions on Industrial Informatics, vol. 12, June 2017.

[12] D. Li, Y. Zhou, G. Hu, and C. J. Spanos, "Optimal sensor configuration and feature selection for AHU fault detection and diagnosis," IEEE Transactions on Industrial Informatics, vol. 12, June 2017.

[13] A. Ashrafian, M. Mirsalim, and M. A. S. Masoum, "Application of a recursive phasor estimation method for adaptive fault component based differential protection of power transformers," IEEE Transactions on Industrial Informatics, vol. 12, June 2017.

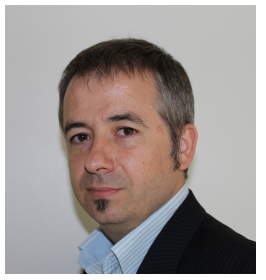

Jose Antonino-Daviu (S04-M08-SM12) received his M.Sc. and Ph. D. degrees in Electrical Engineering, both from the Universitat Politècnica de València, Valencia, Spain in 2000 and 2006, respectively. He worked for IBM, being involved in several international projects. He is currently Associate Professor in the Department of Electrical Engineering of the Universitat Politècnica de València. He has been invited professor in Helsinki University of Technology (Finland) in 2005 and 2007, Michigan State University (USA) in 2010, Korea University (South

Korea) in 2014, Université Claude Bernard Lyon 1 (France) and Coventry University (2016). He is co-author of more than 150 papers published in technical journals and Conference Proceedings. He is also co-author of 1 international patent. He is Associate Editor of IEEE Transactions on Industrial Informatics and Member of the Editorial Board. He was recipient of the IEEE Second Prize Paper Award of the Electric Machines Committee of the IEEE Industry Applications Society (2013). He was also awarded with the Best Paper Award in the conferences IEEE ICEM 2012 and IEEE SDEMPED 2011 and he was awarded with the 'Highly commended recognition' of the IET Innovation Awards in 2014 and in 2016. He has been general co-chair of SDEMPED 2013 and is member of the Steering Committee of IEEE SDEMPED. In 2016, he was awarded with the 'Medal of the Spanish Royal Academy of Engineering'.

In 2016, he was awarded the 'Medal of the Spanish Royal Academy of Engineering' for his contributions in new techniques for predictive maintenance of electric motors.

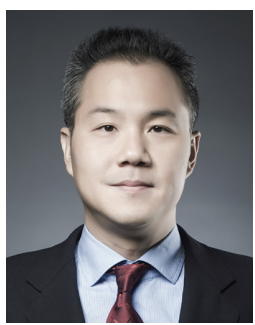

Sang Bin Lee (S'95-M'01-SM'07-F'17) received the B.S. and M.S. degrees from Korea University, Seoul, South Korea, in 1995 and 1997, respectively, and the Ph.D. degree from the Georgia Institute of Technology, Atlanta, GA, USA, in 2001, all in electrical engineering.

From 2001 to 2004, he was at General Electric Global Research Center, Schenectady, NY, USA, where he developed an interlaminar core fault detector for generator stator cores and worked on insulation quality assessment for electric machines. From 2010 to 2011, he was at the Austrian Institute of Technology, Vienna, Austria, where he worked on condition monitoring of permanent magnet synchronous machines. Since 2004, he has been a Professor of electrical engineering with Korea University. His current research interests include protection, monitoring and diagnostics, and analysis of electric machines and drives.

Dr. Lee has received 12 Prize Paper Awards from the IEEE Industry Applications Society (IAS), IEEE Power Engineering Society, Electric Machines Committee of the IEEE IAS, Pulp and Paper Industry Committee of the IEEE IAS, and the Technical Committee on Diagnostics of the IEEE Power Electronics Society. He served as a 2014 - 2016 Distinguished/Prominent Lecturer for the IEEE IAS, and serves an Associate Editor for the IEEE TRANSACTIONS ON INDUSTRY APPLICATIONS for the IEEE IAS Electric Machines Committee.

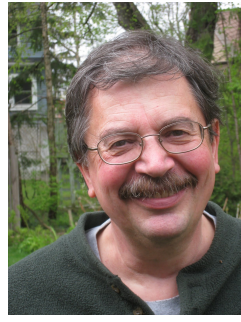

Elias G. Strangas received the Dipl. Eng. degree in Electrical Engineering from the National Technical University of Greece, Athens, Greece and the Ph.D. degree from the University of Pittsburgh, Pittsburgh, PA, USA, in 1980. He was with Schneider Electric Athens, from 1981 to 1983 and the University of Missouri, Rolla, MO, USA, from 1983 to 1986.

Since 1986, he has been with the Department of Electrical and Computer Engineering, Michigan State University, East Lansing, MI, USA, where he heads the Electrical Machines and Drives Laboratory. His research interests include the design and control of electrical machines and drives, and fault prognosis and mitigation of electrical drives. $\mathrm{He}$ is a member of the SDEMPED Steering Committee, the General Chair for SDEMPED 2017, a General co-Chair of ICEM 2018, and the recipient of the IEEE Diagnostics Achievement Award, 2015. 